\title{
Erratum to: $I L 8$ gene as modifier of cystic fibrosis: unraveling the factors which influence clinical variability
}

\author{
Larissa Lazzarini Furlan ${ }^{1}$ Fernando Augusto Lima Marson ${ }^{2,3}$. \\ José Dirceu Ribeiro $^{3}$ - Carmen Sílvia Bertuzzo ${ }^{2}$ João Batista Salomão Junior ${ }^{4}$. \\ Dorotéia Rossi Silva Souza ${ }^{5}$
}

Published online: 3 February 2017

(C) Springer-Verlag Berlin Heidelberg 2017

\section{Erratum to: Hum Genet (2016) 135:881-894 DOI 10.1007/s00439-016-1684-4}

In the online version of the original article, Table 2 is incorrect.

The correct version of Table 2 is given below.

The online version of the original article can be found under doi:10.1007/s00439-016-1684-4.

Fernando Augusto Lima Marson

fernandolimamarson@hotmail.com

1 Medical School of São José do Rio Preto, Avenida Brigadeiro Faria Lima, 5416, Vila São José, São José do Rio Preto, São Paulo CEP 15090-000, Brazil

2 Department of Medical Genetics, School of Medical Sciences, University of Campinas, Rua Tessália Vieira de Camargo, 126, Cidade Universitária Zeferino Vaz, Campinas, São Paulo CEP 13083-887, Brazil

3 Department of Pediatrics, School of Medical Sciences, University of Campinas, Rua Tessália Vieira de Camargo, 126, Cidade Universitária Zeferino Vaz, Campinas, São Paulo CEP 13083-887, Brazil

4 Department of Pediatrics and Department of Pediatrics Pneumology, University Hospital, Medical School of São José do Rio Preto, Avenida Brigadeiro Faria Lima, 5416, Vila São José, São José do Rio Preto, São Paulo CEP 15090-000, Brazil

5 Department of Molecular Biology, Research Center for Biochemistry and Molecular Biology, Medical School of São José do Rio Preto, Avenida Brigadeiro Faria Lima, 5416, Vila São José, São José do Rio Preto, São Paulo CEP 15090-000, Brazil 
Table 2 Descriptive analysis of clinical and laboratory markers in patients with cystic fibrosis

\begin{tabular}{|c|c|}
\hline Variables & Distribution $^{\mathrm{a}}$ \\
\hline Gender (male) & $92 / 186(49.5 \%)$ \\
\hline Ethnicity (Caucasian) & $169 / 180(93.9 \%)$ \\
\hline Age (months) & $\begin{array}{l}169 ; 201.41 \pm 171.98 \\
143(7-932)\end{array}$ \\
\hline Onset of symptoms (months) & $159 ; 38.23 \pm 114.03 ; 3(0-720)$ \\
\hline Diagnosis (months) & $171 ; 87.90 \pm 158.39 ; 20(0-833)$ \\
\hline $\begin{array}{l}\text { Onset of digestive symptoms } \\
\text { (months) }\end{array}$ & $140 ; 41.2 \pm 112.61 ; 3(0-720)$ \\
\hline $\begin{array}{l}\text { Onset of pulmonary symptoms } \\
\text { (months) }\end{array}$ & $156 ; 46.33 \pm 123.64 ; 6(0-720)$ \\
\hline Body mass index & $\begin{array}{l}178 ; 17.28 \pm 4.08 ; 16.28 \\
\quad(6.5-35.67)\end{array}$ \\
\hline Nasal polyposis (presence) & $28 / 162(17.3 \%)$ \\
\hline Diabetes mellitus (presence) & $32 / 164(19.5 \%)$ \\
\hline Osteoporosis (presence) & $25 / 162(15.4 \%)$ \\
\hline $\begin{array}{l}\text { Pancreatic insufficiency } \\
\text { (presence) }\end{array}$ & $130 / 162(80.2 \%)$ \\
\hline Meconium ileus (presence) & $23 / 162(14.2 \%)$ \\
\hline 1st Pseudomonas aeruginosa & $121 ; 103.03 \pm 171.74 ; 31(0-872)$ \\
\hline Mucoid P. aeruginosa (presence) & $74 / 173(42.8 \%)$ \\
\hline $\begin{array}{l}\text { Non-mucoid P. aeruginosa } \\
\text { (presence) }\end{array}$ & $99 / 173(57.2 \%)$ \\
\hline $\begin{array}{l}\text { Achromobacter xylosoxidans } \\
\text { (presence) }\end{array}$ & $17 / 174(9.8 \%)$ \\
\hline Burkolderia cepacia (presence) & $25 / 174(14.4 \%)$ \\
\hline $\begin{array}{l}\text { Staphylococcus aureus } \\
\text { (presence) }\end{array}$ & $131 / 174(75.3 \%)$ \\
\hline $\mathrm{SaO}_{2}$ & $159 ; 94.87 \pm 4.28 ; 96$ (66-99) \\
\hline Bhalla score & $113 ; 8.9 \pm 5.77 ; 8(0-25)$ \\
\hline Kanga score & $118 ; 18.78 \pm 5.82 ; 17.5(10-40)$ \\
\hline Shwachman-Kulczycki score & $143 ; 65.97 \pm 16.78 ; 65(20-95)$ \\
\hline \multicolumn{2}{|l|}{ Spirometry markers ${ }^{\mathrm{b}}$} \\
\hline FVC & $142 ; 72.13 \pm 23.86 ; 77$ (19-126) \\
\hline $\mathrm{FEV}_{1}$ & $141 ; 60.99 \pm 25.75 ; 63(17-116)$ \\
\hline $\mathrm{FEV}_{1} / \mathrm{FVC}$ & $144 ; 79.08 \pm 14.99 ; 81(39-113)$ \\
\hline $\mathrm{FEF}_{25} \%$ & $119 ; 61.28 \pm 31.71 ; 60(7-138)$ \\
\hline $\mathrm{FEF}_{50} \%$ & $119 ; 46.2 \pm 31.25 ; 40(3-126)$ \\
\hline $\mathrm{FEF}_{75} \%$ & $116 ; 36.32 \pm 28.77 ; 27.5(4-142)$ \\
\hline $\mathrm{FEF}_{25-75} \%$ & $140 ; 47.16 \pm 32.51 ; 39(5-150)$ \\
\hline
\end{tabular}

Table 2 continued

\begin{tabular}{ll}
\hline Variables & Distribution $^{\mathrm{a}}$ \\
\hline FEF $_{\text {Max }}$ & $114 ; 75.54 \pm 25.59 ; 73.5$ \\
ERV & $(25-137)$ \\
\hline
\end{tabular}

$\mathrm{SaO}_{2}$ transcutaneous arterial hemoglobin oxygen saturation, $F V C$ forced vital capacity, $F E V_{1}$ forced expiratory volume in $1 \mathrm{~s}$ of $\mathrm{FVC}$, $F E F_{25}$ forced expiratory flow at $25 \%$ of $\mathrm{FVC}, F E F_{50}$ forced expiratory flow at $50 \%$ of $\mathrm{FVC}, F E F_{75}$ forced expiratory flow at $75 \%$ of $\mathrm{FVC}, F E F_{25-75} \%$ average forced expiratory flow between 25 and $75 \%$ of $\mathrm{FVC}, F E F_{\max }$ maximum forced expiratory flow, $E R V$ expiratory reserve volume

${ }^{a}$ The data with categorical distribution are presented as follows: $n$ of variable/ $N$ total (percentage); data with numeric distribution are presented as follows: sample size; mean \pm standard deviation; median (minimum-maximum)

b Spirometry data are shown in percentage of the predicted value according to Polgar and Promadhat (1971), Pereira et al. (2007) and Duarte et al. (2007) equations 